\title{
Pregnancy after Endometriosis: Maternal and Neonatal Outcomes according to the Location of the Disease
}

\author{
Stefano Uccella, MD, PhD ${ }^{1,2}$ Paolo Manzoni, MD ${ }^{3}$ Antonella Cromi, MD, PhD ${ }^{2}$ Nicola Marconi, MD ${ }^{2}$ \\ Baldo Gisone, MD ${ }^{2}$ Andrea Miraglia, MD ${ }^{2}$ Sara Biasoli, MD ${ }^{2}$ Pier Carlo Zorzato, MD ${ }^{1}$ \\ Stefania Ferrari, MD ${ }^{1}$ Gabriele Lanzo, MD ${ }^{1}$ Francesca Bertoli, CNM, MS ${ }^{2}$ Vito Andrea Capozzi, MD ${ }^{4}$ \\ Davide Gallina, MD ${ }^{1}$ Massimo Agosti, MD ${ }^{5}$ Fabio Ghezzi, MD²
}

\footnotetext{
1 Division of Obstetrics and Gynecology, Department of Maternal, Neonatal and Infant Medicine, Nuovo Ospedale degli Infermi, Biella, Italy

2 Department of Obstetrics and Gynecology, Filippo Del Ponte

Hospital, University of Insubria, Varese, Italy

${ }^{3}$ Division of Pediatrics and Neonatology, Department of Maternal and Infant Medicine, Nuovo Ospedale degli Infermi, Biella, Italy

${ }^{4}$ Department of Obstetrics and Gynecology, University of Parma,

Parma, Italy

${ }^{5}$ Department of Neonatology and Neonatal Intensive Care Unit, University of Insubria, Varese, Italy
}

\begin{abstract}
Address for correspondence Stefano Uccella, MD, PhD, Division of Obstetrics and Gynecology, Department of Maternal, Neonatal and Infant Medicine, Nuovo Ospedale degli Infermi, Via dei Ponderanesi, 2-13058, Ponderano, Biella, Italy (e-mail: stefucc@libero.it).
\end{abstract}

Am J Perinatol 2019;36(suppl S2):S91-S98.

\section{Abstract}

\section{Keywords}

- endometriosis

- pregnancy

- deep endometriosis

- endometrioma

- neonatal outcomes

- maternal outcomes

Objective To systematically evaluate pregnancy and labor course, obstetrical complications, and maternal and neonatal outcomes in women with endometriosis, stratifying according to the specific location of the disease.

Study Design We retrospectively analyzed our prospectively maintained obstetrical database from January 2011 to August 2014 to identify all women with a previous histological diagnosis of endometriosis who delivered at our institution (cases). We divided the cases according to the specific location of the disease (deep infiltrating endometriosis, ovarian endometriosis, and peritoneal endometriosis). As controls, we identified all unaffected women who delivered in the year 2013. To avoid the confounding effect of parity, we limited our analysis to nulliparous women.

Results A total of 118 nulliparous women with endometriosis and 1,690 nulliparous controls were identified. Women with endometriosis were significantly older, had a lower body mass index, and had a higher incidence of assisted reproductive technology. The duration of pregnancy was significantly shorter among women with endometriosis. A higher incidence of placenta previa ( 3.4 vs. $0.5 \% ; p=0.006$ ), hypertension (11 vs. $5.9 \% ; p=0.04$ ), cesarean section ( 41.5 vs. $24.2 \% ; p<0.0001$ ), and vacuum delivery ( 10.1 vs. $2.9 \% ; p=0.006$ ) was found in women with endometriosis. Neonatal outcomes were similar between groups. The incidence of placenta previa in patients with deep endometriosis was 11.7 versus $0.5 \%$ among controls $(p<0.0001)$, whereas in women with ovarian and peritoneal endometriosis, it was similar to the controls.
\end{abstract}

Copyright @ 2019 by Thieme Medical Publishers, Inc., 333 Seventh Avenue, New York, NY 10001, USA. Tel: +1(212) 584-4662.
DOI https://doi.org/ 10.1055/s-0039-1692130. ISSN $0735-1631$. 
Conclusion Women with endometriosis have a higher incidence of vacuum delivery, cesarean section, and placenta previa compared with unaffected women. The higher risk of placenta previa is attributable exclusively to women with deep endometriosis. Neonatal outcomes are unaffected by the presence of the disease.

Endometriosis is a common gynecological disease that is defined as the presence of endometrial tissue in ectopic locations; it typically affects women in their fertile age. Three different forms of the disease have been historically described according to the invasiveness and site of the implants: peritoneal disease, ovarian endometriomas, and deep infiltrating endometriosis. ${ }^{1}$ It has been hypothesized that the different anatomical locations may reflect diverse pathogenetic mechanisms and different clinical course. ${ }^{1}$ Regardless of the location of the disease, the estimated prevalence of endometriosis has been concerningly described to be up to $11 \%$ in the general female population, ${ }^{2}$ but it dramatically increases among infertile women. ${ }^{3}$ Although it has been clearly demonstrated that endometriosis reduces fertility, ${ }^{4-7}$ pregnancy among women affected by this disease is becoming increasingly common, thanks to assisted reproductive technology (ART) ${ }^{8}$ and complex surgical eradicative procedures. ${ }^{9-17}$ Despite the rising rates of pregnant women with a previous diagnosis of endometriosis (and in many cases also previous surgical procedures for this condition), information regarding the possible effect of the disease on obstetrical and neonatal outcomes is still scant. Initial reports described an apparently increased risk of preterm birth among women with endometriosis. ${ }^{18-21}$ An intriguing hypothesis linking endometriotic process and preterm delivery regards the possible role of inflammation, with cross-reactions among cytokines, hormones and growth factors. ${ }^{22}$ A recent interesting review of the available literature has concluded that complications of endometriosis during pregnancy are rare and that pregnant women affected by this disease can be reassured on the course of the gestation. ${ }^{23}$ However, a nonnegligible increase in the likelihood of placenta accrete, preterm birth, and aesarean delivery has been described. ${ }^{23-26}$

Unfortunately, most of the available evidence relies either on population-based studies, which used codes of the diagnoses for both the mother and the newborn at the time of discharge from the hospital, or on small retrospective collections of data with conflicting results. Moreover, attention has been given almost exclusively to pregnancy and maternal outcomes, whereas scant data are available on the neonates. At the Department of Obstetrics and Gynecology of the University of Insubria, we have been maintaining for years a detailed prospective collection of perinatal data (regarding both the mother and the newborn) on women who deliver at our institution. With the aid of this valuable tool, we designed this study to systematically evaluate pregnancy and labor course, obstetrical complications, and maternal and neonatal outcomes in women with endometriosis, stratifying according to the specific location of the disease.

\section{Materials and Methods}

The obstetrical database of the Department of Obstetrics and Gynecology of the University of Insubria was queried from January 2011 to August 2014 to identify all women with a previous diagnosis of endometriosis who delivered at our institution (cases). This database is a research quality dataset that is designed and approved for both research and internalaudit purposes, is updated on a regular basis by trained residents, and has thorough and accurate information regarding a patient's history, course of pregnancy, possible obstetrical complications, details of delivery and peripartum period, and neonatal outcomes.

To avoid the possible confounding effect deriving from the inclusion of multiparous women, we focused only on nulliparous women with histologically proven endometriosis. The ascertainment of the diagnosis of endometriosis and the anatomical localization of the disease was conducted as follows: the obstetrical database contains details of medical history of endometriosis, including the site of the lesions and the treatment received (whether surgical only or surgical + pharmacological). For patients previously operated at our institution, a manual search of the operative charts of the patients was performed to confirm the diagnosis, the anatomical site of the disease, the treatment performed, and the rAFS (revised American Fertility Society) score. Patients operated elsewhere were contacted by phone and were asked to provide the operative charts to obtain details of their disease and of the procedures performed. In case of uncertainty or missing histological diagnosis, and/or inaccurate description of the anatomical localization of disease, patients were excluded from the study.

The cases of women with endometriosis were then divided according to the site of the lesions in the following manner: (1) deep infiltrating endometriosis (with or without ovarian and peritoneal localizations), (2) ovarian endometriomas (with or without peritoneal endometriosis), and (3) peritoneal endometriosis only (i.e., patients with only superficial localizations on the peritoneum and no other types of lesions). The control group was represented by all nulliparous women who delivered during the year 2013 but who did not have any history of suspected or confirmed endometriosis. Institutional Review Board approval was obtained for the prospective collection of data, their retrospective analysis, and collection of followup information.

Our analysis focused on the identification of maternal and fetal/neonatal outcomes during pregnancy, in the intrapartum period, and in the postpartum period, comparing all cases of women affected by endometriosis versus controls 
and then stratifying according to the site of the disease. To correct for the possible confounding effect of ART, we also performed a subanalysis including only those patients who conceived spontaneously.

\section{Statistical Analysis}

Statistical analysis was performed using GraphPad Prism version 5.00 for Windows (GraphPad Software, San Diego, CA). Incidence of binomial variables was analyzed for statistical significance using the Fisher exact test. Regarding continuous variables, normality testing (D'Agostino and Pearson test) was performed to determine whether data were sampled from a Gaussian distribution. The Student $t$ test and Mann-Whitney $U$ test were used to compare continuous parametric and nonparametric variables, respectively. A $p$-value of $<0.05$ was considered statistically significant.

\section{Results}

A total of 118 nulliparous women were affected by endometriosis delivered at our institution during the study period and were included in this analysis, and 1,690 patients were selected as controls. The characteristics of the cases (women with endometriosis) and controls (unaffected women) are shown in - Table 1. Women with previously histologically proven endometriosis were significantly older, had a lower body mass index (BMI), and had a higher incidence of ART compared with controls. The duration of pregnancy was significantly shorter among women with endometriosis $(p=0.0002)$, although the difference was only 5 days (39 weeks and 4 days among controls vs. 38 weeks and 6 days among cases). Eighteen (15.3\%) and 9 (7.6\%) patients in the endometriosis group and $194(11.5 \%)$ and $70(4.1 \%)$ in the control group delivered before $37(p=0.24)$ and 34 $(p=0.09)$ weeks of gestation, respectively. The relative risk of delivering before 34 weeks of gestation was 1.81 (95\% confidence interval: $0.95-3.43$ ) in the endometriosis group compared with the controls. A higher incidence of placenta previa ( $3.4 \mathrm{vs.} 0.5 \% p=0.006$ ) and hypertension (11 vs. $5.9 \% ; p=0.04$ ) was found in women affected by endometriosis compared with controls.

- Table 2 reports the maternal and neonatal outcomes at delivery in cases and controls. Women with endometriosis had a lower incidence of spontaneous labor and a higher risk of cesarean section and vaginal vacuum delivery compared with controls. Neonatal outcomes in terms of birthweight, Apgar score at 5 minutes, umbilical artery $\mathrm{pH}$ at birth, incidence of $\mathrm{pH}<7$, and neonatal intensive care unit (NICU) admissions were similar between groups.

- Table 3 reports the comparison between the 64 patients with ovarian endometriosis and the controls. Patients in the ovarian endometriosis group were significantly older and had a lower BMI, a lower median gestational age at delivery, a lower incidence of spontaneous onset of labor, and a higher rate of cesarean section as well as a vacuum delivery compared with controls. The incidence of placenta previa and the neonatal outcomes were similar between groups.

- Table 4 reports the comparison between the 20 women with peritoneal endometriosis and the controls. The age and BMI of the patients were comparable between the two groups. The incidence of vacuum delivery and cesarean section were significantly higher, whereas the likelihood of

Table 1 Characteristics of the cases affected by endometriosis (study group) versus unaffected controls (control group)

\begin{tabular}{|l|l|l|l|}
\hline Parameter & Study group & Control group & $p$-Value \\
\hline No. of patients & 118 & 1,690 & \\
\hline Age & $34(22-45)$ & $31(15-48)$ & $<\mathbf{0 . 0 0 0 1}$ \\
\hline \multicolumn{1}{|c|}{35 years (\%) } & $50(42.4)$ & $434(25.7)$ & $\mathbf{0 . 0 0 1}$ \\
\hline BMI (kg/m ) & $24.71(19.69-32.42)$ & $26.4(17.59-48.87)$ & $\mathbf{0 . 0 4 9}$ \\
\hline $\begin{array}{l}\text { Assisted reproductive } \\
\text { technique (\%) }\end{array}$ & $17(14.4)$ & $100(5.9)$ & $\mathbf{0 . 0 0 1}$ \\
\hline Multiple fetal gestations (\%) & $6(5.1)$ & $65(3.9)$ & 0.46 \\
\hline Gestational age at delivery & $38.9(29.9-42)$ & $39.6(23.3-42.1)$ & $<\mathbf{0 . 0 0 1}$ \\
\hline Maternal weight at delivery (kg) & $70(45.5-98)$ & $72(47-146)$ & 0.08 \\
\hline Smoking habit (\%) & $9(7.6)$ & $97(5.7)$ & 0.41 \\
\hline $\begin{array}{l}\text { Preexisting medical } \\
\text { comorbidities (\%) }\end{array}$ & $1(0.8)$ & $12(0.7)$ & 0.59 \\
\hline Hypertension/preeclampsia (\%) & $13(11)$ & $99(5.9)$ & $\mathbf{0 . 0 4}$ \\
\hline GDM (\%) & $6(5.1)$ & $127(7.5)$ & 0.46 \\
\hline Placenta previa (\%) & $4(3.4)$ & $51(3)$ & $\mathbf{0 . 0 0 6}$ \\
\hline IUGR (\%) & $6(5.1)$ & 0.27 \\
\hline
\end{tabular}

Abbreviations: BMI, body mass index; GDM, gestational diabetes mellitus; IUGR, intrauterine growth restriction. Note: Bold characters highlight statistically significant findings. 
Table 2 Delivery outcomes: patients with endometriosis versus control group

\begin{tabular}{|l|l|l|l|}
\hline Parameter & Study group & Control group & $p$-Value \\
\hline No. of patients & 118 & 1,690 & \\
\hline Spontaneous labor (\%) & $56(47.5)$ & $997(58.9)$ & $\mathbf{0 . 0 1 5}$ \\
\hline Labor induction (\%) & $34(31.4)$ & $452(26.7)$ & 0.67 \\
\hline Failed induction (\%) & $10(8.5)$ & $86(5.1)$ & 0.13 \\
\hline Epidural analgesia in labor (\%) ${ }^{\text {a }}$ & $34 / 89(38.2)$ & $659 / 1,469(44.8)$ & 0.23 \\
\hline Vaginal delivery (\%) & $69(58.5)$ & $1,281(75.8)$ & $<0.0001$ \\
\hline Vacuum delivery (\%) & $7(10.1)$ & $37(2.9)$ & 0.006 \\
\hline CS (\%) & $49(41.5)$ & $409(24.2)$ & $<0.0001$ \\
\hline CS in labor (\%) & $20(16.9)$ & $188(11.1)$ & 0.07 \\
\hline Blood transfusion (\%) & $3(2.5)$ & $36(2.1)$ & 0.74 \\
\hline EBL (mL) & $375(50-3,850)$ & $300(50-3,000)$ & 0.23 \\
\hline Postpartum hemorrhage (\%) & $21(17.8)$ & $413(24.4)$ & 0.051 \\
\hline Postpartum urinary retention (\%) & $1(0.9)$ & $48(2.8)$ & 0.37 \\
\hline Postpartum hospital stay (days) & $3(1-8)$ & $3(0-15)$ & 0.32 \\
\hline Neonatal weight at birth (grams) & $3,105(1,250-4,090)$ & $3,120(400-5,030)$ & 0.19 \\
\hline Apgar at 5 min & $10(4-10)$ & $10(0-10)$ & 0.91 \\
\hline NICU admissions (\%) & $7(5.9)$ & $94(5.6)$ & 0.68 \\
\hline Umbilical artery pH at birth & $7.29(6.97-7.48)$ & $7.27(6.67-7.45)$ & 0.69 \\
\hline
\end{tabular}

Abbreviations: CS, cesarean section; EBL, estimated blood loss; NICU, neonatal intensive care unit.

Note: Bold characters highlight statistically significant findings.

${ }^{a}$ The percentage is calculated on the total number of women who entered labor.

vaginal delivery was significantly lower in patients with peritoneal endometriosis compared with controls; the neonatal outcomes were similar between groups.

- Table 5 shows the comparison between the 34 women with deep endometriosis compared with controls. The patients in the deep endometriosis group had a lower gestational age at birth and a higher incidence of cesarean section, as well as a higher likelihood of hypertensive disorders of pregnancy. The incidence of placenta previa in the group of patients with deep endometriosis was 11.7 versus $0.5 \%$ among controls $(p<0.0001)$. The birthweight, Apgar score at 5 minutes, umbilical artery $\mathrm{pH}$ at birth, incidence of $\mathrm{pH}<7$, and NICU admissions were similar between groups.

These results did not change after exclusion of patients who obtained pregnancy with assisted reproductive techniques.

\section{Discussion}

This study demonstrates that women who deliver after a previous histological diagnosis of endometriosis are leaner, are older, and have a lower likelihood of spontaneous onset of labor and a higher incidence of cesarean section and vacuum delivery compared with patients without endometriosis. We also reported a higher rate of hypertensive disorders of pregnancy and placenta previa in women affected by the disease. The observed increase in the incidence of placenta previa is attributable exclusively to the group of women affected by deep infiltrating endometriosis. It is interesting to note that neonatal outcomes are not affected by the presence and location of endometriosis.

Another interesting finding of our study is the observed tendency (although not significant) toward a higher rate of preterm delivery $<34$ weeks of gestation among women with endometriosis. Even though patients with the disease have a significantly shorter duration of pregnancy, the clinical significance of this shortening appears limited (only 5 days in median) and therefore should not be regarded as a major issue in the everyday clinical practice.

Previous studies have already shown an association between endometriosis and some unfavorable pregnancy outcomes. ${ }^{18-26}$ However, two interesting and comprehensive reviews by Leone Roberti Maggiore et al have highlighted that the risk of complications associated with endometriosis during pregnancy is low. ${ }^{23,24}$ While the available literature suggests that there is an increased risk of placenta previa, particularly in patients with deep endometriosis, pregnant women with endometriosis should be in general reassured regarding the course of their gestation. Few studies, however, have focused on the possible similarities and differences among the different types and locations of endometriosis. ${ }^{27}$ Moreover, while a discrete number of papers have focused on women's health, the literature is devoid of information on the impact of endometriosis on the course of labor and on neonatal outcomes. Thanks to our systematic and thorough collection of data, we were able to 
Table 3 Adnexal endometriosis versus control group

\begin{tabular}{|c|c|c|c|}
\hline Parameter & Adnexal endometriosis & Control group & $p$-Value \\
\hline No. of patients & 64 & 1,690 & \\
\hline Gestational week at delivery & $38.9(30-41.9)$ & 39.6 (range 23.3-42.1) & 0.016 \\
\hline Age & $34(26-44)$ & $31(15-48)$ & $<0.0001$ \\
\hline Spontaneous labor (\%) & $28(43.7)$ & $997(58.9)$ & 0.02 \\
\hline Vaginal delivery (\%) & $40(62.5)$ & $1,281(75.7)$ & 0.02 \\
\hline Vacuum delivery (\%) & $4(10)$ & 37 (2.9) & 0.03 \\
\hline CS (\%) & $24(37.5)$ & $409(24.3)$ & 0.02 \\
\hline CS in labor (\%) & $8(33.3)$ & $188(45.9)$ & 0.29 \\
\hline $\mathrm{BMI}$ & $24.2(19.7-27.9)$ & $26.4(17.5-48.8)$ & 0.002 \\
\hline Smoking habit (\%) & 7 (10.9) & $97(5.7)$ & 0.09 \\
\hline Hypertension/preeclampsia (\%) & $7(10.9)$ & $99(5.9)$ & 0.10 \\
\hline GDM (\%) & $2(3.1)$ & $127(7.5)$ & 0.32 \\
\hline Placenta previa & 0 & $8(0.5)$ & 1.00 \\
\hline $\mathrm{EBL}(\mathrm{mL})$ & $375(100-2,000)$ & $300(50-3,000)$ & 0.18 \\
\hline Postpartum hemorrhage (\%) & $10(15.6)$ & $413(24.4)$ & 0.13 \\
\hline Blood transfusion (\%) & $2(4.6)$ & $36(2.1)$ & 0.64 \\
\hline Postpartum urinary retention & 0 & $48(2.8)$ & 0.41 \\
\hline Postpartum hospital stay (days) & $3(2-7)$ & $3(0-15)$ & 0.47 \\
\hline Neonatal weight at birth & $3,100(1,350-3,960)$ & $3,120(400-5,030)$ & 0.18 \\
\hline Apgar at 5 minutes & $10(5-10)$ & $10(0-10)$ & 0.66 \\
\hline NICU admissions (\%) & $3(4.7)$ & $94(5.6)$ & 0.76 \\
\hline IUGR (\%) & $3(4.6)$ & $51(3)$ & 0.44 \\
\hline Analgesia (\%) & $22 / 40(55)$ & $659 / 1,281(51.4)$ & 0.74 \\
\hline Umbilical artery $\mathrm{pH}$ at birth & $7.3(6.97-7.46)$ & $7.27(6.67-7.45)$ & 0.18 \\
\hline
\end{tabular}

Abbreviations: BMI, body mass index; CS, cesarean section; EBL, estimated blood loss; GDM, gestational diabetes mellitus; NICU, neonatal intensive care unit; IUGR, intrauterine growth restriction.

Note: Bold characters highlight statistically significant findings.

provide consistent results and explore the aspects that have been less investigated.

The higher incidence of cesarean section observed in our study is maintained also after the exclusion of women who underwent ART. The possible explanations for this observation are difficult to find. While the older age and the higher incidence of placenta previa may obviously play a role, it may be hypothesized that the possible coexistence of adenomyosis could be responsible for altered uterine contractility both during and before labor. However, these assumptions should be investigated and proven with specific and personalized research. On the other hand, we observed that there is no detrimental impact of endometriosis on the newborn's health, even in case of deep endometriosis.

It is interesting to note that different subtypes of endometriosis are associated with different pregnancy outcomes. In general, our findings suggest that peritoneal endometriosis should be regarded as the mildest expression of the disease even when considering the course of preg- nancy. On the other hand, it is well known that deep infiltrating endometriosis is the most severe form of the disease, with the worst symptoms and the highest technical difficulty when surgical treatment is to be accomplished. When dealing with pregnancy, deep endometriosis appears as the subtype of disease associated with the poorest outcomes in comparison with ovarian and peritoneal endometriosis.

We acknowledge several limitations of our study. First, the retrospective design may be the source of possible selection, detection and reporting bias. However, we emphasize that our systematic collection of data considerably reduces these possible drawbacks. Another possible limitation of our series is that we did not stratify according to the radicality of the surgery performed for endometriosis, particularly for deep endometriosis. However, it is commonly accepted that the complete removal of the disease during surgical procedures is associated not only with a higher risk of complications but also with a significant improvement in terms of fertility rates. ${ }^{9-11,13}$ Moreover, it is our policy to completely remove 
Table 4 Peritoneal endometriosis versus control group

\begin{tabular}{|l|l|l|l|}
\hline Parameter & Peritoneal endometriosis & Control group & $p$-Value \\
\hline No. of patients & 20 & 1,690 & \\
\hline Gestational age at delivery & $38.8(29.9-42)$ & $39.6(23.3-42.1)$ & 0.16 \\
\hline Multiple fetal gestations (\%) & $1(5)$ & $65(3.9)$ & 0.54 \\
\hline Age (years) & $33.5(26-39)$ & $31(15-48)$ & 1.00 \\
\hline Spontaneous labor (\%) & $9(45)$ & $997(58.9)$ & 0.25 \\
\hline Vaginal delivery (\%) & $10(50)$ & $1,281(75.8)$ & 0.02 \\
\hline Vacuum delivery (\%) & $3(30)$ & $37(2.9)$ & 0.003 \\
\hline CS (\%) & $10(50)$ & $409(24.2)$ & 0.02 \\
\hline CS in labor (\%) & $6(60)$ & $188(45.9)$ & 0.52 \\
\hline BMI & $24.62(20.8-28.4)$ & $26.4(17.5-48.8)$ & 0.5 \\
\hline Smoking habit (\%) & $2(10)$ & $97(5.7)$ & 0.32 \\
\hline Hypertension/preeclampsia (\%) & $1(5)$ & $99(5.9)$ & 1.00 \\
\hline GDM (\%) & $1(5)$ & $127(7.5)$ & 1.00 \\
\hline Placenta previa (\%) & 0 & $8(0.5)$ & 1.00 \\
\hline Estimated blood loss (mL) & $350(50-900)$ & $300(50-3,000)$ & 0.76 \\
\hline Postpartum hemorrhage (\%) & $5(25)$ & $413(24.4)$ & 1.00 \\
\hline Blood transfusion & 0 & $36(2.1)$ & 1.00 \\
\hline Postpartum urinary retention (\%) & $1(5)$ & $48(2.8)$ & 0.44 \\
\hline Postpartum hospital stay (days) & $3(2-4)$ & $3(0-15)$ & 0.41 \\
\hline Neonatal weight (grams) & $3,150(1,250-4,090)$ & $3,120(400-5,030)$ & 0.82 \\
\hline Apgar at 5 min & $10(7-10)$ & $10(0-10)$ & 0.24 \\
\hline NICU admissions (\%) & $1(5)$ & $94(5.6)$ & 1.00 \\
\hline IUGR (\%) & $1(5)$ & $51(3)$ & 0.46 \\
\hline Epidural analgesia in labor (\%) & $5(50)$ & $659 / 1,281(51.4)$ & 1.00 \\
\hline Umbilical artery pH at birth & $7.31(7.11-7.48)$ & $7.27(6.67-7.45)$ & 0.02 \\
\hline
\end{tabular}

Abbreviations: BMI, body mass index; CS, cesarean section; GDM, gestational diabetes mellitus; NICU, neonatal intensive care unit; IUGR, intrauterine growth restriction.

Note: Bold characters highlight statistically significant findings.

Table 5 Deep endometriosis versus control group

\begin{tabular}{|l|l|l|l|}
\hline Parameter & Deep endometriosis & Control group & $p$-Value \\
\hline No. of patients & 34 & 1,690 & \\
\hline Gestational age at delivery & $38.6(30-41.6)$ & $39.6(23.3-42.1)$ & 0.002 \\
\hline Age (years) & $33.5(22-45)$ & $31(15-48)$ & 0.09 \\
\hline Spontaneous labor (\%) & $15(44.1)$ & $997(58.9)$ & 0.11 \\
\hline Vaginal delivery (\%) & $19(55.8)$ & $1,281(75.7)$ & $\mathbf{0 . 0 1 3}$ \\
\hline Vacuum delivery (\%) & 0 & $37(2.9)$ & 1.000 \\
\hline CS (\%) & $15(44.2)$ & $409(24.3)$ & $\mathbf{0 . 0 1 3}$ \\
\hline CS in labor (\%) & $6(40)$ & $188(45.9)$ & 1.000 \\
\hline BMI & $27(23.5-32.4)$ & $26.4(17.5-48.8)$ & 0.54 \\
\hline Smoking habit (\%) & 0 & $97(5.7)$ & 0.25 \\
\hline Hypertension/preeclampsia (\%) & $5(14.7)$ & $99(5.9)$ & 0.03 \\
\hline GDM (\%) & $2(5.8)$ & $127(7.5)$ & 0.19 \\
\hline
\end{tabular}


Table 5 (Continued)

\begin{tabular}{|l|l|l|l|}
\hline Parameter & Deep endometriosis & Control group & $p$-Value \\
\hline Placenta previa (\%) & $4(11.7)$ & $8(0.5)$ & $<\mathbf{0 . 0 0 0 1}$ \\
\hline Estimated blood loss (mL) & $400(50-3,850)$ & $300(50-3,000)$ & 0.84 \\
\hline Postpartum hemorrhage (\%) & $6(17.6)$ & $413(24.4)$ & 0.42 \\
\hline Blood transfusion (\%) & $1(2.9)$ & $36(2.1)$ & 0.52 \\
\hline Episiotomy & & & \\
\hline Postpartum urinary retention & 0 & $48(2.8)$ & 1.00 \\
\hline Postpartum hospital stay (days) & $3(1-8)$ & $3(0-15)$ & 0.003 \\
\hline Neonatal weight at birth (grams) & $3,080(1,530-3,760)$ & $3,120(400-5,030)$ & 0.42 \\
\hline Apgar at 5 min & $10(4-10)$ & $10(0-10)$ & 0.23 \\
\hline NICU admissions (\%) & 94() & 0.42 \\
\hline IUGR (\%) & $3(8.8)$ & $51(3)$ & 0.28 \\
\hline Epidural analgesia in labor (\%) & $7 / 19(36.8)$ & $659 / 1,281(51.4)$ & 1.00 \\
\hline Umbilical artery pH at birth & $7.26(7.01-7.49)$ & $7.27(6.67-7.45)$ & 0.66 \\
\hline
\end{tabular}

Abbreviations: BMI, body mass index; CS, cesarean section; GDM, gestational diabetes mellitus; NICU, neonatal intensive care unit; IUGR, intrauterine growth restriction.

Note: Bold characters highlight statistically significant findings.

the disease when surgery is indicated. As a consequence, we assume that almost all the patients included in this study had optimal removal of endometriosis at the time of surgery before the onset of pregnancy.

In conclusion, our analysis provides useful data on an appropriate and moderately optimistic counseling to pregnant women previously operated for endometriosis. Our findings show that although patients with deep endometriosis have a considerably higher risk of placenta previa $(\sim 1$ out of 10), the overall outcomes of pregnancy and in particular the neonatal outcomes are in line with those of unaffected women.

\section{Funding}

None.

\section{Conflict of Interest}

None declared.

\section{References}

1 Nisolle M, Donnez J. Peritoneal endometriosis, ovarian endometriosis, and adenomyotic nodules of the rectovaginal septum are three different entities. Fertil Steril 1997;68(04):585-596

2 Buck Louis GM, Hediger ML, Peterson CM, et al; ENDO Study Working Group. Incidence of endometriosis by study population and diagnostic method: the ENDO study. Fertil Steril 2011;96 (02):360-365

3 Macer ML, Taylor HS. Endometriosis and infertility: a review of the pathogenesis and treatment of endometriosis-associated infertility. Obstet Gynecol Clin North Am 2012;39(04):535-549

4 Harb HM, Gallos ID, Chu J, Harb M, Coomarasamy A. The effect of endometriosis on in vitro fertilisation outcome: a systematic review and meta-analysis. BJOG 2013;120(11):1308-1320

5 Falconer H. Pregnancy outcomes in women with endometriosis. Semin Reprod Med 2013;31(02):178-182
6 Practice Committee of the American Society for Reproductive Medicine. Endometriosis and infertility. Fertil Steril 2006;86(05, Suppl Suppl 1):S156-S160

7 Legendre G, Catala L, Morinière C, et al. Relationship between ovarian cysts and infertility: what surgery and when? Fertil Steril 2014;101(03):608-614

8 Prefumo F, Rossi AC. Endometriosis, endometrioma, and ART results: current understanding and recommended practices. Best Pract Res Clin Obstet Gynaecol 2018;51:34-40

9 Uccella S, Gisone B, Serati M, et al. Functional outcomes of nervesparing laparoscopic eradication of deep infiltrating endometriosis: a prospective analysis using validated questionnaires. Arch Gynecol Obstet 2018;298(03):639-647

10 Uccella S, Marconi N, Casarin J, et al. Impact of endometriosis on surgical outcomes and complications of total laparoscopic hysterectomy. Arch Gynecol Obstet 2016;294(04):771-778

11 Uccella S, Cromi A, Agosti M, et al. Fertility rates, course of pregnancy and perinatal outcomes after laparoscopic ureterolysis for deep endometriosis: a long-term follow-up study. J Obstet Gynaecol 2016;36(06):800-805

12 Rausei S, Sambucci D, Spampatti S, et al. Laparoscopic treatment of deep infiltrating endometriosis: results of the combined laparoscopic gynecologic and colorectal surgery. Surg Endosc 2015;29(10):2904-2909

13 Uccella S, Cromi A, Casarin J, et al. Laparoscopy for ureteral endometriosis: surgical details, long-term follow-up, and fertility outcomes. Fertil Steril 2014;102(01):160-166

14 Ceccaroni M, Ceccarello M, Caleffi G, et al. Total laparoscopic ureteroneocystostomy for ureteral endometriosis: a single-center experience of 160 Consecutive Patients. J Minim Invasive Gynecol 2019;26(01):78-86

15 Ceccaroni M, Clarizia R, Roviglione G, Ruffo G. Neuro-anatomy of the posterior parametrium and surgical considerations for a nerve-sparing approach in radical pelvic surgery. Surg Endosc 2013;27(11):4386-4394

16 Ceccaroni M, Clarizia R, Bruni F, et al. Nerve-sparing laparoscopic eradication of deep endometriosis with segmental rectal and parametrial resection: the Negrar method. A singlecenter, prospective, clinical trial. Surg Endosc 2012;26(07): 2029-2045 
17 Iosca S, Lumia D, Bracchi E, et al. Multislice computed tomography with colon water distension (MSCT-c) in the study of intestinal and ureteral endometriosis. Clin Imaging 2013;37(06):1061-1068

18 Ueda Y, Enomoto T, Miyatake T, et al. A retrospective analysis of ovarian endometriosis during pregnancy. Fertil Steril 2010;94 (01):78-84

19 Brosens I, Brosens JJ, Fusi L, Al-Sabbagh M, Kuroda K, Benagiano G. Risks of adverse pregnancy outcome in endometriosis. Fertil Steril 2012;98(01):30-35

20 Stephansson O, Kieler H, Granath F, Falconer H. Endometriosis, assisted reproduction technology, and risk of adverse pregnancy outcome. Hum Reprod 2009;24(09):2341-2347

21 Fernando S, Breheny S, Jaques AM, Halliday JL, Baker G, Healy D. Preterm birth, ovarian endometriomata, and assisted reproduction technologies. Fertil Steril 2009;91(02):325-330

22 Petraglia F, Arcuri F, de Ziegler D, Chapron C. Inflammation: a link between endometriosis and preterm birth. Fertil Steril 2012;98 (01):36-40
23 Leone Roberti Maggiore U, Ferrero S, Mangili G, et al. A systematic review on endometriosis during pregnancy: diagnosis, misdiagnosis, complications and outcomes. Hum Reprod Update 2016;22 (01):70-103

24 Leone Roberti Maggiore U, Inversetti A, Schimberni M, Viganò P, Giorgione V, Candiani M. Obstetrical complications of endometriosis, particularly deep endometriosis. Fertil Steril 2017;108 (06):895-912

25 Zullo F, Spagnolo E, Saccone G, et al. Endometriosis and obstetrics complications: a systematic review and meta-analysis. Fertil Steril 2017;108(04):667-672

26 Chen I, Lalani S, Xie RH, Shen M, Singh SS, Wen SW. Association between surgically diagnosed endometriosis and adverse pregnancy outcomes. Fertil Steril 2018;109(01):142-147

27 Exacoustos C, Lauriola I, Lazzeri L, De Felice G, Zupi E. Complications during pregnancy and delivery in women with untreated rectovaginal deep infiltrating endometriosis. Fertil Steril 2016; 106(05):1129-1135 